ARTICLE OPEN

\title{
Tuning magnetic confinement of spin-triplet superconductivity
}

\author{
Wen-Chen Lin $\mathbb{C}^{1,7}$, Daniel J. Campbell ${ }^{1,7}$, Sheng Ran ${ }^{1,2,3}$, I-Lin Liu $\mathbb{C}^{1,2,3}$, Hyunsoo Kim $\mathbb{C}^{1}$, Andriy H. Nevidomskyy ${ }^{4}$, David Graf $\mathbb{1}^{5}$,
} Nicholas P. Butch $\left(\mathbb{D}^{1,2}\right.$ and Johnpierre Paglione $\mathbb{D}^{1,3,6 凶}$

Electrical magnetoresistance and tunnel diode oscillator measurements were performed under external magnetic fields up to $41 \mathrm{~T}$ applied along the crystallographic $b$ axis (hard axis) of UTe $e_{2}$ as a function of temperature and applied pressures up to $18.8 \mathrm{kbar}$. In this work, we track the field-induced first-order transition between superconducting and magnetic field-polarized phases as a function of applied pressure, showing suppression of the transition with increasing pressure until the demise of superconductivity near $16 \mathrm{kbar}$ and the appearance of a pressure-induced ferromagnetic-like ground state that is distinct from the field-polarized phase and stable at zero field. Together with evidence for the evolution of a second superconducting phase and its upper critical field with pressure, we examine the confinement of superconductivity by two orthogonal magnetic phases and the implications for understanding the boundaries of triplet superconductivity.

npj Quantum Materials (2020)5:68; https://doi.org/10.1038/s41535-020-00270-w

\section{INTRODUCTION}

Previous work on uranium-based compounds, such as $\mathrm{UGe}_{2}$, URhGe, and UCoGe, has unearthed a rich interplay between superconductivity and ferromagnetism in this family of materials ${ }^{1}$, with suggestions that ferromagnetic spin fluctuations can act to enhance pairing ${ }^{2}$. The recent discovery of superconductivity in $\mathrm{UTe}_{2}$ has drawn strong attention owing to a fascinating list of properties-including the absence of magnetic order at ambient pressure $^{3}$, Kondo correlations, and extremely high upper critical fields ${ }^{4}$ - that have led to proposals of spin-triplet pairing ${ }^{4-7}$, and a chiral order parameter ${ }^{8,9}$.

In addition, at least two forms of re-entrant superconductivity have been observed in high magnetic fields, including one that extends the low-field superconducting phase upon precise field alignment along the crystallographic $b$ axis ${ }^{10}$, and an extreme high-field phase that onsets in pulsed magnetic fields above the paramagnetic normal state at angles tilted away from the $b$ axis ${ }^{11}$.

Applied pressure has also been shown to greatly increase the superconducting critical temperature $T_{\mathrm{c}}$ in $\mathrm{UTe}{ }_{2}^{12,13}$, from $1.6 \mathrm{~K}$ to nearly double that value near $10 \mathrm{kbar}$, and to induce a second superconducting phase above a few $\mathrm{kbar}^{13}$. Upon further pressure increase, evidence of suppression of the Kondo energy scale leads to an abrupt disappearance of superconductivity and a transition to a ferromagnetic phase ${ }^{12}$. Together with the ambient pressure magnetic field-induced phenomena ${ }^{10,11,14,15}$, the axes of the magnetic field, temperature, and pressure provide for a very rich and interesting phase space in this system. One of the key questions is in regard to the field-polarized (FP) phase that appears to truncate superconductivity at $34.5 \mathrm{~T}$ under proper $b$-axis field alignment ${ }^{10,11}$, in particular regarding the nature of the coupling of the two phases and whether superconductivity could persist to even higher fields in the absence of the competing FP phase. The relation between the FP phase and the pressureinduced magnetic phase, which also competes with superconductivity ${ }^{11}$, is similarly not yet fully understood.
In this work, we perform magnetoresistance (MR) and tunnel diode oscillator (TDO) measurements under both high hydrostatic pressures $P$ and high magnetic fields $H$ along the crystallographic $b$ axis to explore the $(H, T, P)$ phase diagram. We find that the FP phase that interrupts superconductivity at ambient pressure is strengthened with increasing pressure, so as to suppress the transition field until there is no trace of superconductivity down to $0.4 \mathrm{~K}$ above $16 \mathrm{kbar}$. At higher pressures, we find evidence of a distinct magnetic phase that appears to be ferromagnetic in nature and is also bordered by the FP phase at finite fields. Together with previous observations at ambient pressure, these results suggest a spectrum of magnetic interactions in $\mathrm{UTe}_{2}$ and a multifaceted ground state sensitive to several physical tuning parameters.

\section{RESULTS AND DISCUSSION}

Experimental results

The magnetic field response of electrical resistance $R$ at low pressures is similar to previous results at ambient pressure, which showed that the superconducting state persists up to the FP phase transition $H^{*}$ of nearly $35 \mathrm{~T}$ for $H \| b$, and re-entrant behavior can be observed near $T_{\mathrm{c}}$ for a slight misalignment of the field ${ }^{10}$. While it is not presently known why the $b$-axis alignment is crucial, it is thought that alignment of the applied field and fluctuating moments plays an important role ${ }^{10,11}$. As shown in Fig. 1a, application of $4 \mathrm{kbar}$ of pressure reduces the cut-off field $H^{*}$ to $30 \mathrm{~T}$ at $0.38 \mathrm{~K}\left(T_{\mathrm{c}}=1.7 \mathrm{~K}\right.$ without applied field) but retains the very sharp transition to the FP state, above which a negative MR ensues. Upon temperature increase, a re-entrant feature emerges below $H^{*}$ similar to the previous reports ${ }^{10}$ but only above about $1.3 \mathrm{~K}$, indicating either nearly perfect alignment along the $b$ axis or reduced sensitivity to field angle at finite pressures.

Upon further pressure increase, $T_{\mathrm{C}}$ increases as previously shown ${ }^{12,13}$, up to $2.6 \mathrm{~K}$ and $2.8 \mathrm{~K}$ at $8.5 \mathrm{kbar}$ and $14 \mathrm{kbar}$, respectively. However, $H^{*}$ is continuously reduced through this

\footnotetext{
${ }^{1}$ Maryland Quantum Materials Center, Department of Physics, University of Maryland, College Park, MD 20742, USA. ${ }^{2}$ NIST Center for Neutron Research, National Institute of Standards and Technology, Gaithersburg, MD 20899, USA. ${ }^{3}$ Department of Materials Science and Engineering, University of Maryland, College Park, MD 20742, USA. ${ }^{4}$ Department of Physics and Astronomy, Rice University, Houston, TX 77005, USA. ${ }^{5}$ National High Magnetic Field Laboratory, Florida State University, Tallahassee, FL 32313, USA. ${ }^{6}$ Canadian Institute for Advanced Research, Toronto, Ontario M5G 1Z8, Canada. ${ }^{7}$ These authors contributed equally: Wen-Chen Lin, Daniel J. Campbell. ${ }^{\circledR}$ email: paglione@umd.edu
} 

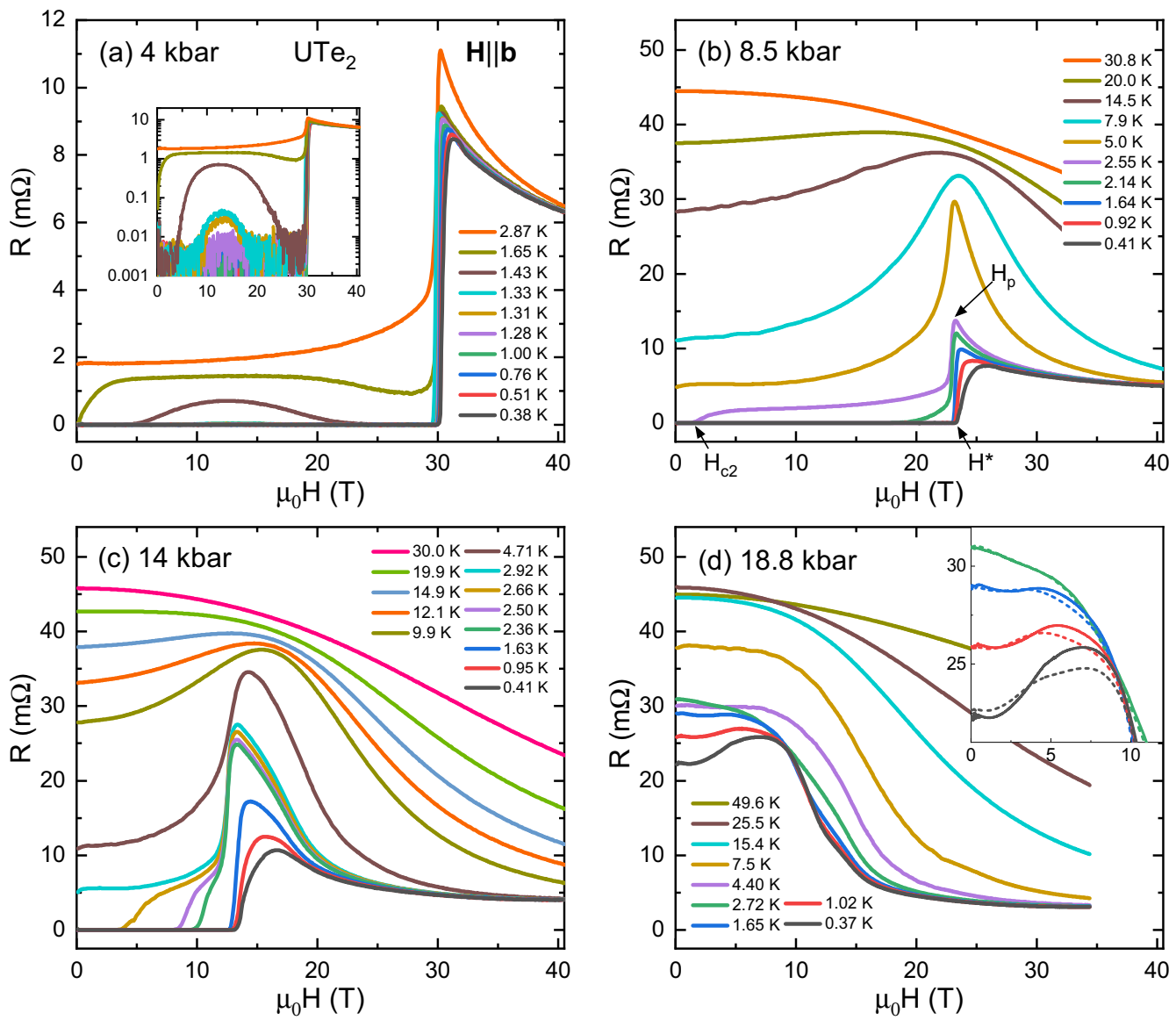

Fig. 1 Magnetoresistance of $\mathrm{UTe}_{2}$ under applied pressures. Magnetoresistance of a $\mathrm{UTe}_{2}$ single crystal with current applied along crystallographic $a$ axis and magnetic fields applied along the $b$ axis under applied pressures of (a) $4 \mathrm{kbar}$, (b) $8.5 \mathrm{kbar}$, (c) $14 \mathrm{kbar}$, and (d) $18.8 \mathrm{kbar}$. Inset of (a) shows a semilog plot of magnetoresistance at $4 \mathrm{kbar}$, highlighting re-entrant superconductivity. In panel (b)), the applied field at the resistance peak $\left(H_{\mathrm{p}}\right)$ and the critical field $\left(H_{\mathrm{c} 2}\right)$ are labeled on the violet curve as an example. The cut-off field $\left(H^{*}\right)$ at base temperature is also labeled. Inset of (d) presents a zoom in the range where hysteresis is observed via distinct upsweep (solid lines) and downsweep (dashed lines) curves.

range and changes in character. As shown in Fig. $1 \mathrm{~b}$ and $\mathrm{c}$, at higher pressures $H^{*}$ and $H_{c 2}$ dissociate, beginning as a single sudden rise with a broadened peak (denoted $H_{\mathrm{p}}$ ) in resistance at $0.4 \mathrm{~K}$ that becomes better-defined upon increasing from lowest temperature, before separating into two distinct transitions at higher temperatures. Interestingly, the transition is the sharpest when the $H_{\mathrm{c} 2}$ transition separates from $H^{*}$ and moves down in the field. Further, the coupled transitions slightly decrease in the field until about $2 \mathrm{~K}$, above which the resistive $H_{\mathrm{c} 2}$ continues to decrease while $H^{*}$ stalls (e.g., at about $12 \mathrm{~T}$ for $14 \mathrm{kbar}$ ) until washing out above $\sim 20 \mathrm{~K}$. This indicates a strong coupling between the two transitions that is weakened both on pressure increase and temperature increase, despite the first-order nature of the FP phase. At $18.8 \mathrm{kbar}$, shown in Fig. 1d, where no superconducting phase is observed down to $0.37 \mathrm{~K}$, the sharp feature associated with $H^{*}$ is gone, and only a broad maximum in $R$ remains near $8 \mathrm{~T}$. Around this feature, we observed hysteresis loops at low temperature as shown in the inset (see Supplementary Fig. 6 for hysteresis loops under both positive and negative fields). Together with the evidence from previous pressure experiments identifying similar hysteretic behavior ${ }^{12}$, we believe there is a ferromagnetic-like ground state that evolves from zero temperature and zero magnetic field, and, similar to superconductivity at lower pressures, is truncated by the FP phase and therefore distinct from that ground state. The crossover from the FM-like ground state to the FP phase is also supported by the drop of resistance at around $10 \mathrm{~T}$.
Figure 2 presents the frequency variation $\Delta f$ in the TDO signal, where a minus sign has been applied to the frequency following convention. The frequency variations reflect the changes in magnetic susceptibility and therefore are sensitive to the anomalies that cannot be captured through transport measurements in the zero-resistance regime. In addition to a sharp rise in $\Delta f$ at $H^{*}$, which corresponds to a diamagnetic to paramagnetic transition, and changes in slope consistent with the re-entrant behavior mentioned above (see Supplementary Fig. 4), there is another feature in the $4 \mathrm{kbar}$ data within the superconducting state observable at lower fields. At temperatures below $1 \mathrm{~K}, \Delta f$ initially increases with field before abruptly transitioning to a constant above a characteristic field $H_{\mathrm{c} 2(2)}$, and finally jumping at the $H^{*}$ transition. As the temperature is increased, $H_{\mathrm{c2}(2)}$ decreases in field value until it vanishes above $T_{c}$, tracing out an apparent phase boundary within the superconducting state. As shown in Fig. 3, the path of $H_{\mathrm{c} 2(2)}$ merges with the zero-field critical temperature of the second superconducting phase "SC2" discovered by ac calorimetry measurements ${ }^{13}$. As shown in Fig. 3a, these data identify SC2 as having a distinct $H_{\mathrm{c} 2}(T)$-phase boundary from the higher- $T_{\mathrm{C}}$ "SC1" phase, with a zero-temperature upper critical field of $\sim 11 \mathrm{~T}$ at $4 \mathrm{kbar}$. Upon further pressure increase, the $H_{\mathrm{c} 2(2)}$ transition is suppressed in field, tracing out a reduced SC2 phase boundary (see Supplementary Fig. 3) that is absent by 14 kbar. In essence, it appears that the SC2 phase is suppressed more rapidly than the SC1 phase, which will provide insight into the distinction between each phase ${ }^{16}$. 

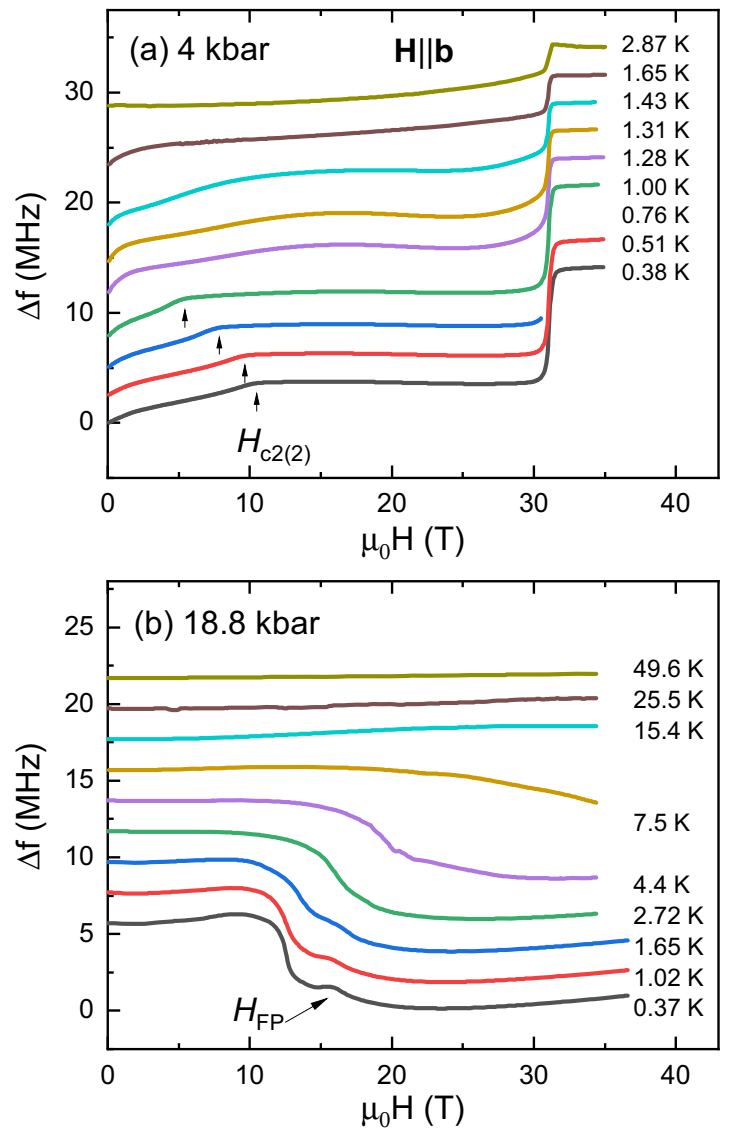

Fig. 2 Field evolution of magnetic susceptibility of $\mathrm{UTe}_{2}$ under applied pressures. Tunnel diode oscillator (TDO) frequency variation of $\mathrm{UTe}_{2}$ single crystal as a function of magnetic fields applied along the crystallographic $b$ axis, under applied pressures of (a) $4 \mathrm{kbar}$ and (b) $18.8 \mathrm{kbar}$. Transitions involving the SC2 superconducting phase are labeled as $H_{\mathrm{c} 2(2)}$ in panel (a), and crossovers to the field-polarized phases (see text) labeled as $H_{\text {FP }}$ in panel (b). All curves are vertically shifted for presentation.

In contrast to the abrupt increase of $\Delta f$ upon crossing $H^{*}$ into the FP phase at lower pressures, the TDO signal exhibits a qualitatively different response in the high-pressure regime where superconductivity is completely suppressed. As shown in Fig. 2b, at $18.8 \mathrm{kbar} \Delta f$ is almost field independent on increasing fields at $0.37 \mathrm{~K}$ until an abrupt drop occurs near $12 \mathrm{~T}$. This drop reflects the decrease of skin depth, which can be confirmed by comparing with the decrease of resistance in our transport results. However, at slightly higher fields, we observe a small peak in $\Delta f$ that does not match any observable feature in transport measurements. This peak suggests a metamagnetic transition at $H_{\mathrm{FP}}(=15.5 \mathrm{~T}$ at $0.37 \mathrm{~K})$, indicating a crossover toward the FP phase.

Phase diagrams and GL theory

Compiling this data, we summarize the observed features and phase boundaries in both resistance and TDO measurements in Fig. 3. We identify five phases: two superconducting phases (labeled SC1 and SC2), the normal phase (labeled N), the FP phase, and the FM phase, which is only observed at $18.8 \mathrm{kbar}$. The first three phase diagrams $(4,8.5$, and 14 kbar) show a smooth growth of the FP phase with pressure and the emergence of a more conventional (i.e., rounded) $H-T$ boundary of the SC1 superconducting phase. In fact, the observable evolution of $H_{\mathrm{c} 2}(T)$ at 8.5 and $14 \mathrm{kbar}$ indicates a putative $H_{\mathrm{c} 2}(0)$ critical point that would end within the FP phase were it not cut off by $H^{*}$.
We estimate these fields to be $72 \mathrm{~T}$ and $55 \mathrm{~T}$ for $8.5 \mathrm{kbar}$ and 14 kbar, respectively (see Supplementary Fig. 5). In this pressure range, where the putative $H_{\mathrm{c} 2}(0)$ scale becomes comparable to the FP scale $H^{*}$, there are clear indications of an influence on the shape of the FP transition as noted above, despite its first-order nature (cf. hysteresis observed at base temperature shown in Fig. 3a, inset). Tracking the resistance peak $H_{\mathrm{p}}$ to fields above $H^{*}$ traces a nonmonotonic curve that, when below $T_{c}$, mimics the extension of $H_{\mathrm{c} 2}(T)$ of the $\mathrm{SC} 1$ phase, again suggesting an intimate correlation between the two phases. This is corroborated by the fact that at $18.8 \mathrm{kbar}$, when superconductivity is completely suppressed, the onset of the FP phase shows a more conventional monotonic evolution with increasing field and temperature.

In an effort to explain the qualitative features of the phase diagram, we consider the phenomenological Ginzburg-Landau (GL) theory describing the superconducting order parameter $\eta$. For simplicity, we shall consider $\eta$ to be single-component, relegating to the Supplementary Note the consideration of a multi-component order parameter proposed theoretically for $\mathrm{UTe}_{2}{ }^{16,17}$ and corroborated by the recent specific heat measurements ${ }^{16}$. The free energy consists of three parts: $F=F_{\mathrm{sc}}[\eta]+$ $F_{m}[\mathbf{M}]+F_{c}[\eta, \mathbf{M}]$, with the first term describing the superconducting order parameter in the applied field ${ }^{18}$ :

$F_{\mathrm{sc}}[\eta]=a(T)|\eta|^{2}+\frac{\beta}{2}|\eta|^{4}+K_{i j}\left(D_{i} \eta\right)^{*}\left(D_{j} \eta\right)+\frac{B^{2}}{8 \pi}$,

with $D_{i}=-i \nabla_{i}+\frac{2 \pi}{\Phi_{0}} A_{i}$ denoting the covariant derivative in terms of the vector potential $\mathbf{A}$ and $\Phi_{0}=h c / 2 e$ the quantum of the magnetic flux, where $K_{i j}=\operatorname{diag}\left\{K_{x}, K_{y}, K_{z}\right\}$ is the effective mass tensor in the orthorhombic crystal, $K_{i}^{-1}=2 m_{i}$. The simplest way, in which the superconducting order parameter couples to the field-induced microscopic magnetization $\mathbf{M}$, is via the biquadratic interaction $F_{c}=g \mathbf{M}^{2}|\eta|^{2}$, where the internal magnetic field $\mathbf{B} / \mu_{0}=$ $\mathbf{M}+\mathbf{H}$. The metamagnetic transition is described by the Landau theory of magnetization with a negative quartic term $(u, v>0)$ :

$F_{m}[\mathbf{M}]=\frac{\mathbf{M}^{2}}{2 \chi(P, T)}+\frac{u}{4} \mathbf{M}^{4}-\frac{v}{6} \mathbf{M}^{6}-\mathbf{H} \cdot \mathbf{M}$.

Taking the field $\mathbf{H} \| \hat{b}$, and hence $\mathbf{A}=(H z, 0,0)$, we minimize the $G L$ free energy to obtain the linearized gap equation of the form

$-K_{z} \frac{\mathrm{d}^{2} \eta}{\mathrm{d} z^{2}}+K_{x}\left(\frac{2 \pi H}{\Phi_{0}}\right)^{2} z^{2} \eta-a_{0} \frac{\left(T_{\mathrm{c}}-T\right)}{T_{\mathrm{c}}} \eta+g \mathbf{M}^{2} \eta=0$,

resulting in the eigenvalue spectrum similar to the problem of Landau levels for a particle in magnetic field ${ }^{19}$ :

$\hbar \omega_{c}\left(n+\frac{1}{2}\right)=a_{0}\left(\frac{T_{c}-T}{T_{c}}\right)-g \mathbf{M}^{2}(T)$,

with the cyclotron frequency given by $\omega_{c}=2 e H \sqrt{K_{x} K_{z}} / c$. The upper critical field $H_{c 2}^{\prime}(T)$ is then determined from the lowest eigenvalue above:

$H_{\mathrm{c} 2}^{\prime}(T)=H_{0}\left[\frac{T_{\mathrm{c}}-T}{T_{\mathrm{c}}}-\frac{g}{a_{0}} M^{2}\left(H_{\mathrm{c} 2}\right)\right]$,

where $H_{0}=-\left.T_{\mathrm{c}} \frac{\mathrm{d} H_{\mathrm{c} 2}}{\mathrm{~d} T}\right|_{T_{\mathrm{c}}}$ is related to the slope of $H_{\mathrm{c} 2}$ at $T_{\mathrm{c}}$ in the absence of magnetization and $a_{0}=\frac{\hbar^{2}}{2 m \xi_{0}}$ is expressed in terms of the correlation length. The upshot of Eq. (5) is that the upper critical field is reduced from its bare value by the presence of the magnetization $M$. The latter is a function of the magnetic field, $M$ $(H)$, to be determined from Eq. (2), and while its value depends on the phenomenological coefficients of the Landau theory, qualitatively, the metamagnetic transition results in a sudden increase of $M$ at $H^{*}$ (by $\Delta M \approx 0.6 \mu_{B}$ at $H^{*}=34 \mathrm{~T}$ at ambient pressure ${ }^{10}$ ). This then drives $H_{\mathrm{c} 2}^{\prime}$ down according to Eq. (5) and pins the upper critical field at the metamagnetic transition, explaining the sudden disappearance of superconductivity at the field $H^{*}$ that marks the 

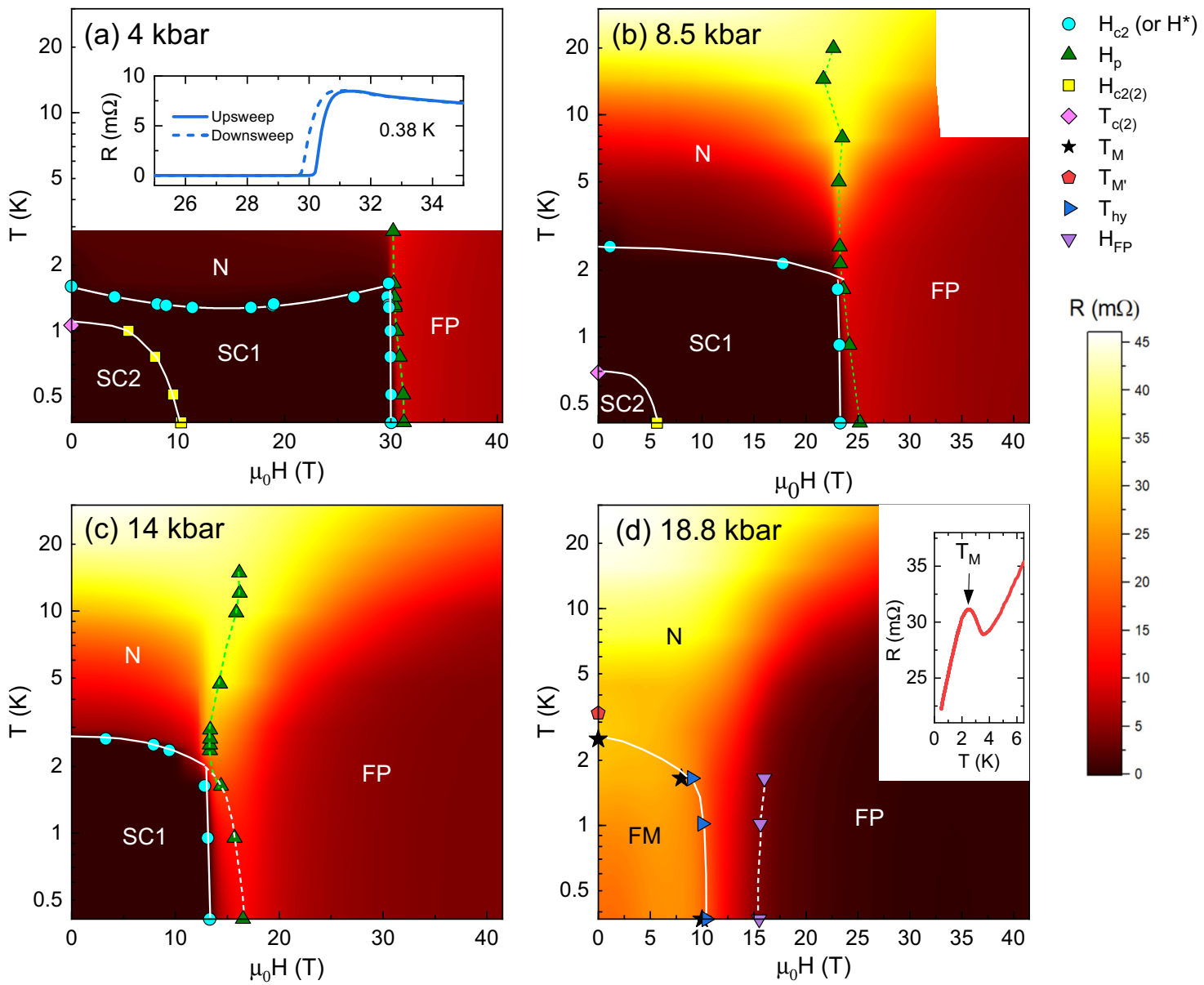

Fig. 3 Pressure evolution of magnetic field-temperature phase diagram of UTe 2 . Evolution of the magnetic field-temperature phase diagram of UTe 2 as a function of pressure for fields applied along the crystallographic $b$ axis, with phase boundaries of superconducting (SC1 and SC2), normal (N), field-polarized (FP), and ferromagnetic (FM) phases determined by resistance and tunnel diode oscillator (TDO) data, and concomitant variations in resistance shown by background color contours. The inset of panel (a) shows the upsweep and downsweep of magnetoresistance around the metamagnetic transition. In panels $(\mathbf{a}-\mathbf{c})$, the cyan circles indicate the $T_{\mathrm{c}}$ transition into the SC1 superconducting phase obtained by field sweeps that are determined by zero-resistance criteria, and the green triangles label the position $H_{\mathrm{p}}$ of the peak in magnetoresistance. Yellow squares in panels $(\mathbf{a}, \mathbf{b})$ indicate critical field $H_{\mathrm{c} 2(2)}$ of the superconducting phase $\mathrm{SC2}$ based on TDO measurements (cf. Fig. 2a), with pink diamonds indicating critical temperature $T_{c(2)}$ obtained from ref. ${ }^{13}$. In panel (d), the purple downward triangles label the crossover to the field-polarized state $H_{\mathrm{FP}}$ identified in TDO measurements (cf. Fig. $2 \mathrm{~b}$ ) while the blue rightward triangles label the demise of hysteresis $H_{\text {hy }}$ in transport measurements. The black star identifies the transition $T_{\mathrm{M}}$ observed in the resistance temperature dependence (panel $\mathbf{d}$, inset) while the red pentagon indicates the same transition measured in ref. ${ }^{13}$.

onset of the FP phase in Fig. 4c. Note that the above analysis focuses on the orbital effect of the applied magnetic field, since SC in $\mathrm{UTe}_{2}$ is not Pauli-limited, presumably due to the equal-spin pairing nature of the pairing ${ }^{17}$.

It is worth discussing the value of $H_{\mathrm{c} 2}$, which is of the order $H_{\mathrm{c} 2}$ $\sim 30 \mathrm{~T}$ at low $T$ and ambient pressure, much higher than would normally be inferred from $T_{c} \sim 2 \mathrm{~K}$. While the analysis of the linearized GL equation above only applies in the vicinity of $T_{\mathrm{c}}$ and cannot, strictly speaking, be used to infer the value of $H_{\mathrm{c} 2}(0)$ at zero temperature, the celebrated Werthamer-Helfand-Hohenberg theory ${ }^{20}$ establishes a proportionality between the value of $H_{c 2}(0)$ and the value $H_{0}$ in Eq. (5). We shall therefore use

$H_{0}=\frac{\Phi_{0}}{2 \pi \hbar^{2}} \frac{a_{0}}{\sqrt{K_{x} K_{z}}} \equiv \frac{\Phi_{0}}{2 \pi \hbar^{2}} a_{0} m^{*}$

as a proxy for the upper critical field $H_{\mathrm{c} 2}(0)\left(\Phi_{0}=h c / 2 e\right.$ is the flux quantum). We see that the role of the effective mass is played by $m^{*}=\left(K_{x} K_{z}\right)^{-1 / 2} \propto \sqrt{m_{a} m_{c}}$ and this helps explain the high observed value of $H_{\mathrm{c} 2}$ in $\mathrm{UTe}_{2}$, as we show below. The key point is the quasi-two-dimensional nature of the Fermi surface sheets parallel to the $c$-axis, established by ARPES $^{21}$ and ab initio calculations ${ }^{17,22}$, which can be approximated by writing the dispersion as

$\varepsilon_{\mathbf{k}}=\frac{\hbar^{2}}{2 m_{a b}}\left[\left(k_{a}-\frac{\pi}{a}\right)^{2}+\left(k_{b}-\frac{\pi}{b}\right)^{2}\right]-2 T_{c} \cos \left(k_{c} d\right)-\mu$,

where we have taken the in-plane mass to be isotropic for simplicity: $m_{a}=m_{b}=m_{a b}$, and $T_{c}$ denotes the interlayer hopping strength along the $c$ axis ( $d$ is the unit cell height). It follows that the carrier mass along $k_{c}$ can be approximated by $m_{c}=\hbar^{2} /\left(2 T_{c} d^{2}\right)$, which in turn means that the effective mass entering Eq. (6), $m^{*} \propto \sqrt{m_{a b} /\left(T_{c} d^{2}\right)}$. Smaller magnitude of the interlayer hopping $T_{\mathrm{c}}$ thus results in a higher value of $H_{0}$ in Eqs. (5) and (6). A more rigorous treatment based on the Green's function formalism reaches a qualitatively similar conclusion: $H_{\mathrm{c} 2}(0) \propto 1 / T_{\mathrm{c}}{ }^{23}$, thus explaining the high values of $\mathrm{H}_{\mathrm{c} 2}$ in $\mathrm{UTe}_{2}$ due to the quasi-twodimensional nature of the Fermi surfaces. The key finding of the present study is that $H_{\mathrm{c} 2}$ is in fact limited from above by the metamagnetic transition at field $H^{*}$, showcased by Eq. (5).

Focusing on the evolution of the ground state of $\mathrm{UTe}_{2}$ with field and pressure (i.e., at our base temperature of $\sim 0.4 \mathrm{~K}$ ), we present 

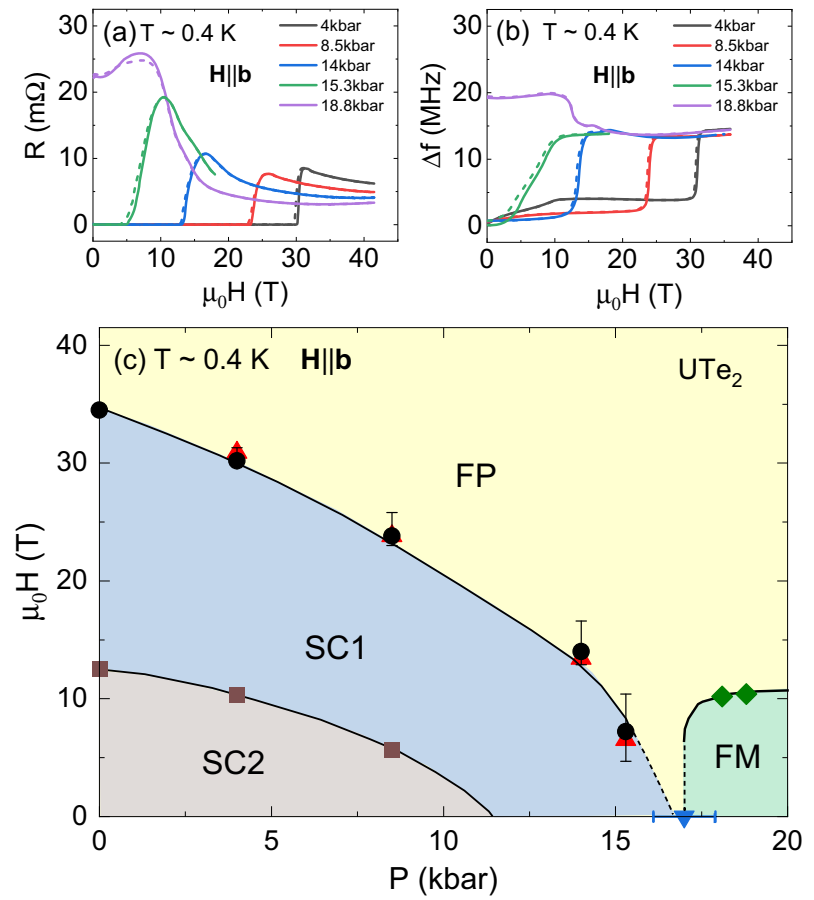

Fig. 4 Ground-state phase diagram of $\mathrm{UTe}_{2}$ under applied field and pressure. Ground-state evolution of superconducting (SC1 and $\mathrm{SC} 2$ ), field-polarized (FP), and ferromagnetic (FM) phases in $\mathrm{UTe}_{2}$ as a function of applied pressure and magnetic field applied along the crystallographic $b$ axis. Panels (a) and (b) present resistance and tunnel diode oscillator (TDO) frequency variation, respectively, as functions of applied field at a fixed base temperature of the measurements. Both upsweeps (solid lines) and downsweeps (dashed lines) are plotted, indicating notable hysteresis. Note that in (b), all data are measured by a standard low-temperature-tuned TDO circuit, while the $15.3 \mathrm{kbar}$ data were obtained using a room temperature-tuned circuit, and is therefore vertically scaled by a factor of 22 for comparison (see Supplementary Figs. 2 and 3c for more $15.3 \mathrm{kbar}$ data). The resultant phase diagram at base temperature is presented in panel (c), where the phase boundary between SC1 and FP phases is determined by midpoints of resistance transitions (black circles, using an average of upsweep and downsweep curves) and TDO transitions (red triangles), with error bars indicating the width of transitions. Brown squares indicate the phase boundary of SC2 based on kinks in TDO frequency, and green diamonds indicate the magnetic transition determined from the resistance measurements. The blue upside-down triangle labels the critical pressure $\left(P_{\mathrm{c}}\right)$ where the superconductivity demises. Zeropressure and zero-field data points are obtained from refs. ${ }^{11,13}$ respectively. All lines are guides to the eye.

summary plots of the resistance and TDO data as well as the ground-state field-pressure phase diagram in Fig. 4. As shown, the field boundaries of both SC1 and SC2 superconducting phases decrease monotonically with increasing pressure. However, we point out that, while the boundary of SC2 appears to be an uninterrupted upper critical field, that of SC1 is in fact the cut-off field $H^{*}$. It follows from Eq. (5) that this cut-off field is reduced compared to the putative $H_{\mathrm{c} 2}$, which would lie at higher fields if it were derived from an orbital-limited model without taking metamagnetic transition into account.

While the $T_{\mathrm{C}}$ of $\mathrm{SC} 1$ increases with pressure, the cutoff imposed by $H^{*}$ introduces difficulty in determining whether its putative $H_{\mathrm{c} 2}$ would also first increase with pressure. On the contrary, the unobstructed view of $H_{\mathrm{c} 2}$ for SC2 shows a decrease with increasing pressure that is indeed consistent with the suggested decrease of the lower $T_{c}$ transition observed in zero-field specific heat measurements ${ }^{13}$.
Between 15.3 and $18.8 \mathrm{kbar}$, the $H^{*}$ cutoff is completely suppressed and the FM phase onsets. While it is difficult to obtain a continuous measure of the pressure evolution through that transition, the hysteresis in transport measurements is consistent with the low-field FM phase being the true magnetic ground state of the system, separate from the FP phase. The crossover toward the FP phase under field is entirely natural from the Landau theory perspective, since the external magnetic field is conjugate to the FM order parameter $\mathbf{M}$ in Eq. (2), and the metamagnetic crossover at field $H_{M}$ leads to a step-like increase in the magnetization, resulting in a small peak in TDO results.

This crossover boundary between the FM and FP phases appears much less sensitive to pressure for $P>P_{c}$ as evidenced by the minimal change in field value between 18.1 and $18.8 \mathrm{kbar}$. Because the experimental pressure cannot be tuned continuously, it is difficult to extract the behavior of the crossover boundary at $P_{c}$. However, the previously observed discontinuity between the FM and SC1 phases as a function of pressure ${ }^{12}$ suggests that the FP phase should extend down to zero field at a critical point of $P_{c} \sim 17 \mathrm{kbar}$, exactly where previous zero-field work has shown an abrupt cutoff of $T_{\mathrm{c}}$ and the onset of a non-superconducting phase $^{13}$. This is different from the case of uranium-based ferromagnetic superconductors $\left(\mathrm{UGe}_{2}, \mathrm{URhGe}, \mathrm{UCoGe}\right)$, where the superconductivity coexists with ferromagnetism. The distinctive behavior of $\mathrm{UTe}_{2}$ is likely owing to a unique nature of its spin fluctuation spectrum, which may also benefit from reduced dimensions at high fields ${ }^{23}$. In any case, as a nearly ferromagnetic superconductor, $\mathrm{UTe}_{2}$ provides a unique platform for future investigation of the interplay between superconductivity and magnetic phases.

In summary, we have explored the pressure evolution of multiple superconducting and multiple magnetic phases of $\mathrm{UTe}_{2}$ as a function of applied pressures and magnetic fields applied along the crystallographic $b$ axis, where superconductivity is known to extend to the highest fields. The field-induced metamagnetic transition results in a field-polarized phase which cuts off superconductivity prematurely, as explained by a phenomenological Ginzburg-Landau theory. Under increasing pressure, the superconducting phase eventually becomes completely suppressed, at the critical pressure where we observe an onset of a distinct ferromagnetic-like ground state.

\section{METHODS}

\section{Measurements}

Single crystals of $\mathrm{UTe}_{2}$ were synthesized by the chemical vapor transport method as described previously ${ }^{4}$. The crystal structure of $\mathrm{UTe}_{2}$ is orthorhombic and centrosymmetric, and the magnetic easy axis is the $a$ axis. Experimental measurements were conducted at the DC Field Facility of the National High Magnetic Field Laboratory (NHMFL) in Tallahassee, Florida, using a 41 T resistive magnet with a helium-3 cryostat. Resistance and magnetic susceptibility measurements were performed simultaneously on two individual samples from the same batch positioned in a non-magnetic piston-cylinder pressure cell. The pressure medium was Daphne 7575 oil, and the pressure was calibrated at low temperatures by measuring the fluorescence wavelength of ruby, which has a known temperature and pressure dependence ${ }^{24,25}$. The TDO technique uses an LC oscillator circuit biased by a tunnel diode whose resonant frequency is determined by the values of $L C$ components, with the inductance $L$ given by a coil that contains the sample under study; the change of its magnetic properties results in a change in resonant frequency proportional to the magnetic susceptibility of the sample. Although not quantitative, the TDO measurement is indeed sensitive to the sample's magnetic response within the superconducting state where the sample resistance is zero ${ }^{26-28}$. Both the current direction for the standard four-wire resistance measurements and the probing field generated by the TDO coil are along the crystallographic $a$ axis (easy axis). The applied dc magnetic field was applied along the $b$ axis (hard axis) for both samples (see Supplementary Fig. 1). 


\section{DATA AVAILABILITY}

The data that support the findings of this study are available from the corresponding author upon reasonable request.

Received: 24 March 2020; Accepted: 9 September 2020;

Published online: 25 September 2020

\section{REFERENCES}

1. Aoki, D., Ishida, K. \& Flouquet, J. Review of U-based ferromagnetic superconductors: Comparison between UGe 2 , URhGe, and UCoGe. J. Phys. Soc. Jpn. 88, 022001 (2019).

2. Mineev, V. P. Reentrant superconductivity in URhGe. Phys. Rev. B 91, 014506 (2015).

3. Sundar, S. et al. Coexistence of ferromagnetic fluctuations and superconductivity in the actinide superconductor UTe 2 . Phys. Rev. B 100, 140502 (2019).

4. Ran, S. et al. Nearly ferromagnetic spin-triplet superconductivity. Science $\mathbf{3 6 5}$, 684-687 (2019).

5. Aoki, D. et al. Unconventional superconductivity in heavy fermion $\mathrm{UTe}_{2}$. J. Phys. Soc. Jpn. 88, 043702 (2019).

6. Metz, T. et al. Point-node gap structure of the spin-triplet superconductor $\mathrm{UTe}_{2}$. Phys. Rev. B 100, 220504 (2019).

7. Nakamine, G. et al. Superconducting properties of heavy fermion $\mathrm{UTe}_{2}$ revealed by 125 Te-nuclear magnetic resonance. J. Phys. Soc. Jpn. 88, 113703 (2019).

8. Bae, S. et al. Anomalous normal fluid response in a chiral superconductor. Preprint at https://arxiv.org/abs/1909.09032 (2019).

9. Jiao, L. et al. Chiral superconductivity in heavy-fermion metal UTe 2 . Nature 579, 523-527 (2020).

10. Knebel, G. et al. Field-reentrant superconductivity close to a metamagnetic transition in the heavy-fermion superconductor UTe 2 . J. Phys. Soc. Jpn. 88, 063707 (2019).

11. Ran, S. et al. Extreme magnetic field-boosted superconductivity. Nat. Phys. 15, 1250-1254 (2019).

12. Ran, S. et al. Enhancement and reentrance of spin triplet superconductivity in $\mathrm{UTe}_{2}$ under pressure. Phys. Rev. B 101, 140503 (2020).

13. Braithwaite, D. et al. Multiple superconducting phases in a nearly ferromagnetic system. Commun. Phys. 2, 147 (2019).

14. Knafo, W. et al. Magnetic-field-induced phenomena in the paramagnetic superconductor UTe 2 . J. Phys. Soc. Jpn. 88, 063705 (2019).

15. Miyake, A. et al. Metamagnetic transition in heavy fermion superconductor $\mathrm{UTe}_{2}$. J. Phys. Soc. Jpn. 88, 063706 (2019).

16. Hayes, I. M. et al. Weyl superconductivity in UTe 2 . Preprint at https://arxiv.org/abs/ 2002.02539 (2020).

17. Nevidomskyy, A. H. Stability of a nonunitary triplet pairing on the border of magnetism in $\mathrm{UTe}_{2}$. Preprint at https://arxiv.org/abs/2001.02699 (2020).

18. Mineev, V. P. \& Samokhin, K. V. Introduction to Unconventional Superconductivity (CRC Press, 1999).

19. Tinkham, M. Introduction to Superconductivity (Dover publications, 2004).

20. Werthamer, N. R., Helfand, E. \& Hohenberg, P. C. Temperature and purity dependence of the superconducting critical field, $H_{c 2}$. III. Electron spin and spinorbit effects. Phys. Rev. 147, 295-302 (1966).

21. Miao, L. et al. Low energy band structure and symmetries of $\mathrm{UTe}_{2}$ from angleresolved photoemission spectroscopy. Phys. Rev. Lett. 124, 076401 (2020).

22. Xu, Y., Sheng, Y. \& Yang, Y.-F. Quasi-two-dimensional fermi surfaces and unitary spin-triplet pairing in the heavy fermion superconductor UTe 2 . Phys. Rev. Lett. 123, 217002 (2019).

23. Mineev, V. P. Reentrant Superconductivity in UTe 2 . Jetp. Lett. 111, 715-719 (2020).

24. Piermarini, G. J., Block, S., Barnett, J. D. \& Forman, R. A. Calibration of the pressure dependence of the $\mathrm{R} 1$ ruby fluorescence line to 195 kbar. J. Appl. Phys. 46, 2774-2780 (1975).

25. Ragan, D. D., Gustavsen, R. \& Schiferl, D. Calibration of the ruby R 1 and R 2 fluorescence shifts as a function of temperature from 0 to 600 k. J. Appl. Phys. 72, 5539-5544 (1992).
26. Kim, H., Sung, N. H., Cho, B. K., Tanatar, M. A. \& Prozorov, R. Magnetic penetration depth in single crystals of $\mathrm{SrPd}_{2} \mathrm{Ge}_{2}$ superconductor. Phys. Rev. B 87, 094515 (2013).

27. Cho, K. et al. Anisotropic upper critical field and possible Fulde-Ferrel-LarkinOvchinnikov state in the stoichiometric pnictide superconductor LiFeAs. Phys. Rev. B 83, 060502 (2011).

28. Prommapan, P. et al. Magnetic-field-dependent pinning potential in LiFeAs superconductor from its Campbell penetration depth. Phys. Rev. B 84, 060509 (2011).

\section{ACKNOWLEDGEMENTS}

We thank H.-K. Wu and Y.-T. Hsu for useful discussions. This work was performed at the National High Magnetic Field Laboratory, which is supported by the National Science Foundation Cooperative Agreement No. DMR-1644779 and the State of Florida. Research at the University of Maryland was supported by AFOSR grant no. FA9550-14-1-0332, NSF grant no. DMR-1905891, the Gordon and Betty Moore Foundation's EPiQS Initiative through grant no. GBMF9071, NIST, and the Maryland Quantum Materials Center. D.J.C. acknowledges the support of the Anne G. Wylie Dissertation Fellowship. A.H.N. acknowledges the support from the Robert A. Welch Foundation grant C-1818 and the National Science Foundation grant no. DMR1917511.

\section{AUTHOR CONTRIBUTIONS}

W.-C.L. and D.J.C. are co-first authors. W.-C.L., D.J.C., and J.P. conceived and designed

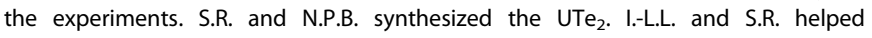
characterize the samples. W.-C.L., D.J.C., and D.G. performed the measurements. A.H.N. provided the theoretical analysis. W.-C.L., D.J.C., H.K., A.H.N., N.P.B., and J.P. analyzed the data and wrote the paper.

\section{COMPETING INTERESTS}

The authors declare no competing interests.

\section{ADDITIONAL INFORMATION}

Supplementary information is available for this paper at https://doi.org/10.1038/ s41535-020-00270-w.

Correspondence and requests for materials should be addressed to J.P.

Reprints and permission information is available at http://www.nature.com/ reprints

Publisher's note Springer Nature remains neutral with regard to jurisdictional claims in published maps and institutional affiliations.

(i) Open Access This article is licensed under a Creative Commons adaptation, distribution and reproduction in any medium or format, as long as you give appropriate credit to the original author(s) and the source, provide a link to the Creative Commons license, and indicate if changes were made. The images or other third party material in this article are included in the article's Creative Commons license, unless indicated otherwise in a credit line to the material. If material is not included in the article's Creative Commons license and your intended use is not permitted by statutory regulation or exceeds the permitted use, you will need to obtain permission directly from the copyright holder. To view a copy of this license, visit http://creativecommons. org/licenses/by/4.0/.

(c) The Author(s) 2020 